\title{
Methodology for the Simulation of Industrial Processes Using Embedded Systems
}

\author{
Henry Montaña Quintero ${ }^{1}$, Vicente Reyes Mozo ${ }^{2}$, Holman Montiel Ariza ${ }^{3}$ \\ Universidad Distrital Francisco José de Caldas, Facultad Tecnológica \\ Cll 68 D Bis A Sur No. 49F - 70, Bogotá D.C., Colombia \\ ${ }^{1}$ hmontanaq@udistrital.edu.co, ${ }^{2}$ jvreyesm@udistrital.edu.co \\ ${ }^{3}$ hmontiela@udistrital.edu.co
}

\begin{abstract}
The instrumentation that is implemented in industrial processes currently has a large number of functions, due to the robustness and intolerance to failures that must show during its continuous operation. This characteristic raises the costs of these elements by reducing their possibility of being acquired by institutions dedicated to teaching instrumentation and control, such as; universities or technical education institutes. This problem limits the access of students to adequate technical training, which increases the gap between the industry and the academy. Some partial solutions are implemented through different software that allows an approach to industrial-type instruments through simulations; however, these do not yet contemplate all the variables that can be presented in practice. An alternative solution to this topic is proposed in this article, which shows a methodology to emulate the behavior of different actuators that can be had in practice using embedded systems.
\end{abstract}

Keyword - Embedded system, approximate model, sensor and actuator.

\section{INTRODUCTION}

The industrial instrumentation existing in the market is mostly used in the manufacturing lines of large companies due to its high cost; this characteristic reduces the access to this type of instruments to educational institutions, which does not allow the subjects of the technical subjects to be updated reducing the effectiveness of students in practical life [1-2].

Some partial solutions allow simulating certain behaviors of different elements of industrial type, such as: valves, motors or pneumatic actuators. However, the libraries of the elements that make up this type of simulator represent linear or ideal behavior, reducing the accuracy of the results obtained during the simulation. In addition, in some cases the authors use generic elements that are not homologous in a real implementation [35].

These generic elements are usually modeled by mathematical expressions, which represent the dynamic behavior of the instrument. These behaviors are usually determined by ordinary differential equations, under certain criteria or restrictions among which are: the location, the operating time, the opposition to a magnitude, among others. One way to complement the analyzes by means of differential equations, is based on obtaining technical information from the manufacturers and thus parameterizing the elements that make up the control system or the instrument to be modeled [6-8].

These approximate models determine the stability of a system or take the system to a stable state by applying different control techniques, such as: Zieger Nichols, Cohen and Coon or the locus of the roots. These techniques have proven highly effective in achieving a system that reaches an expected value, however, they are limited to systems that work in continuous time and in practice, many control systems are based on digital controllers [9-10]. These controllers work with discrete signals and under different parameters than those estimated with continuous time models. This reduces the effectiveness of the analytical methods, since, when converting the parameters estimated in a continuous time system to a discrete time system, the margin of error between the original model and the one that is intended to lead to the practice [10].

By increasing the margin of error, the effectiveness of the controller is reduced, which does not allow the methods implemented analytically or simulated to become a tool for analyzing industrial processes by students [11]. Consequently, this paper describes a partial solution to the limitation that occurs in the approach to industrial models in an analytical manner, from the generation of different signals of a process through a digital system. The advantage of this strategy is that it allows generalizing different industrial instruments; therefore, it is flexible enough to allow the academic community to have an experience closer to the handling of industrial variables in academic spaces. The characteristics described above are organized as follows: Sections 2 and 3 define the basic parameters for the treatment of discrete signals and their relationship with a control system. Section 4 presents the results obtained by implementing the simulation technique proposed in this article. 


\section{METHODOLOGY}

The control systems have a particular way to be treated by simulations, in several cases it is get from a practical element to an analytical model from polynomial approximations that are made by numerical methods, among which are: interpolations, ARIMA methods or transformations in discrete time. Among them, ARIMA models are used in economics due to their degree of precision and are defined as an integrated self-regressive model of moving average (ARIMA is an English acronym that means autoregressive integrated moving average). This statistical model is based on variations that allow finding patterns towards the future, based on regressions of statistical data. They have found great acceptance in the control area, since they allow modeling system dynamics through time series. In other words, predictions of data are given by past data and unlike conventional methods it is based on independent variables [8-9].

The ARIMA models are composed of a certain number of coefficients and a certain number of regressions. It is usually represented as ARIMA ( $p, d, q)$, where the parameters $p, d, q$ are positive integers that indicate the order of the components of the model where $\mathrm{p}$ represents the auto-regressive component, $d$ represents the integrated component and $\mathrm{q}$ the moving average. When one wants to eliminate some of the components, simply matches the component to zero (0) and an approximate mathematical model that represents the ARIMA models is presented in equation $1[8-9]$.

$$
Y_{t}=-\left(\Delta^{d} Y_{t}-Y_{t}\right)+\emptyset_{0}+\sum_{i=1}^{p} \emptyset_{i} \Delta^{d} Y_{t-i}-\sum_{i=1}^{q} \theta_{i} \varepsilon_{t-i}+\varepsilon_{t}
$$

In this group of terms, $\mathrm{d}$ represents the differences that convert the original data series into a series of stationary data, that is, that part of a mathematical expression is constructed from the original data. The variable $\varnothing$ represents the author-regressive part and $\theta$ the moving parts of the model. Taking into account that $\varepsilon$ represents the error and is responsible for feedback to the model to correct the coefficients [9].

Thanks to the approximations that allow making this type of models, sensors and actuators can be represented analytically. Of which it is possible to mention that a sensor allows capturing a magnitude of the environment, since, this type of elements is sensitive to the magnitude for which it was designed. These types of elements are usually conditioned using different electronic circuits. These circuits allow the sensor to have a linear response to a physical variable, which makes them ideal for performing different types of industrial applications among which they are: digital thermometers, anemometers or tachometers [14-15].

By conditioning the sensors, different control strategies can be implemented in a system, because the variation of the error is also linear. This feature accommodates the controllers of type P (Proportional), PI (Proportional-Integral) or PID (Proportional, Integral and Derivative), which make an adjustment in the output from the input error and are the most used to industrial level, since they require few parameters to perform a successful configuration as in models based on neural networks [12] or fuzzy logic [13].

\section{DESIGN AND IMPLEMENTATION}

The simulation of an industrial type element in an embedded system was made from experimental data. These data allow finding a mathematical expression approximate to the model of the system, which is known as system identification. In this case, the identification of a tank system belonging to the process control system T5552 was carried out.

The procedure consists in making the connection of a signal generating element and another measuring element to the actuators of the T5552 system, which when activated generates a disturbance that allows the filling of the tanks (see Fig. 1). As the tanks are filled, a NI6291 National Instruments data acquisition card, which functions as an interface, records the system's behavior through a level sensor with a sampling time of $100 \mathrm{~ms}$ (see Table I).

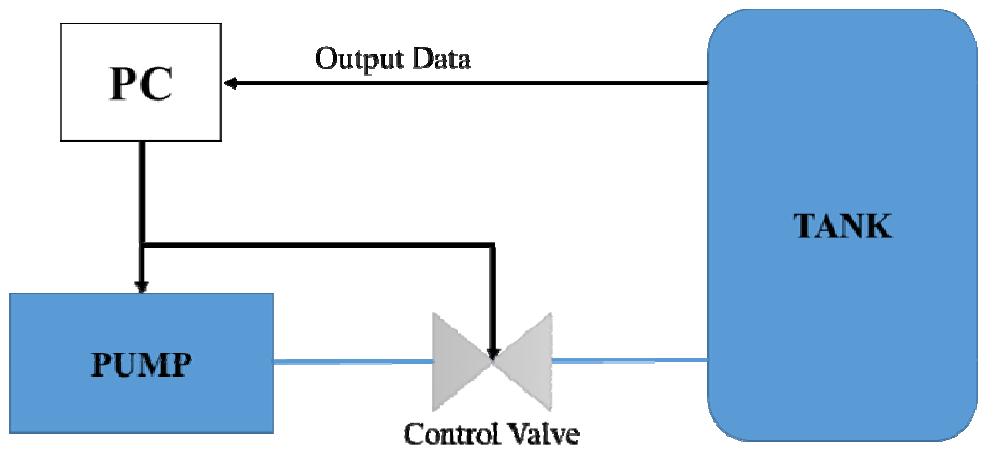

Fig 1. Assembled structure. 
As shown in Fig. 1, the computer is responsible for making the variations of the valve and switching the pump status, in order to obtain different samples of the behavior of the sensors in the process during operation. Attempting to obtain a variation of the input regarding the output without damaging the pump, it was decided to turn on the pump during the registration process and to alternate the status of the proportional valve. Since the proportional valve and the sensor are normalized, both work in a range of 4-20mA, which was taken as reference to generate the input signals (Table I, column 5) and thus have data in the output (Table I, column 3). The identification was made using math software, whose resulting equation was programmed into the PSLO 5LP microcontroller using the strategy of conversion to difference equations from the ARIMA model found by the software.

TABLE I. Segment of the recorded data.

\begin{tabular}{|c|c|c|c|c|}
\hline Time (s) & Level $(\mathbf{c m})$ & Level $(\mathbf{m A})$ & Pump status & Valve Opening (mA) \\
\hline $\mathbf{0}$ & -0.172332397 & 5.429478924 & OFF & 4 \\
\hline $\mathbf{0 . 1}$ & 0.316505384 & 5.500582601 & OFF & 4 \\
\hline $\mathbf{0 . 2}$ & 0.480203745 & 5.524393272 & OFF & 4 \\
\hline $\mathbf{0 . 3}$ & 0.549016571 & 5.53440241 & OFF & 4 \\
\hline $\mathbf{0 . 4}$ & 0.501738584 & 5.527525612 & OFF & 4 \\
\hline $\mathbf{0 . 5}$ & 1.279232955 & 5.640615703 & OFF & 4 \\
\hline $\mathbf{0 . 6}$ & 0.300502868 & 5.498254963 & OFF & 4 \\
\hline
\end{tabular}

The equation in difference is programmed as the decomposition of several retainers of order 1, which allow replicating the filling behavior of the tank which is controlled using a PID. The PID selected was the one incorporated in the PLC (programmable logic controller) of the SIEMENS S7 300 range, that is, the embedded system works like the plant emulator and the PLC is an external mechanism that allows to control the process variable (Level) emulated by the embedded system (see Fig. 2).

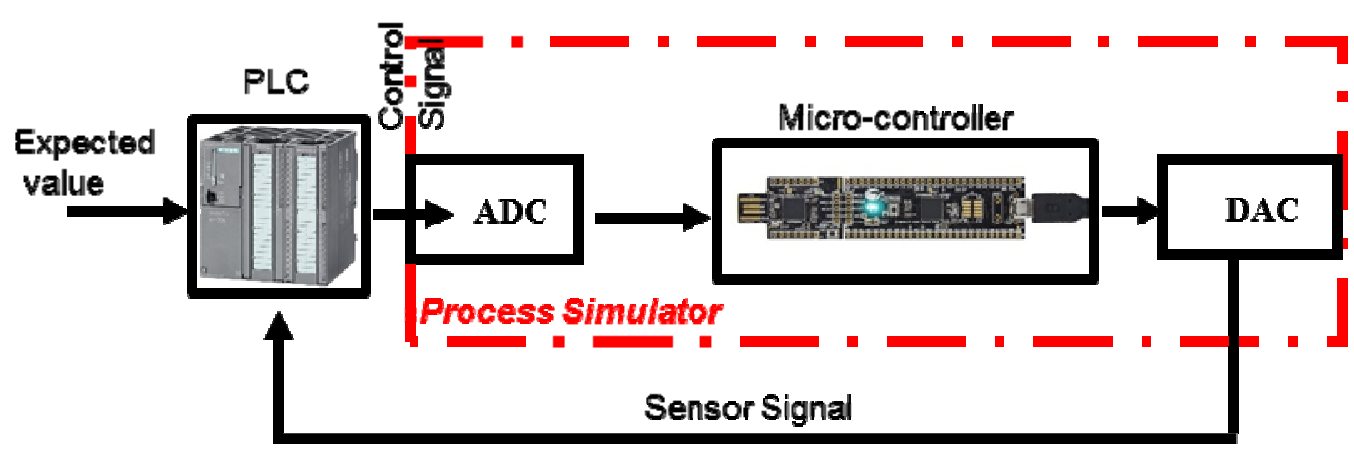

Fig 2. Connection of the simulator with the PLC.

\section{RESULTS AND DISCUSSION}

Initially, the identification of the system was carried out, which allowed finding a mathematical representation with a correlation index close to $98 \%$. This index represents the fidelity of the mathematical expression compared to the sampling made of the system to be simulated (see Table II).

Next, the coefficients found using the software of the canonical representation of the ARIMA model (Equation 2) allow to construct the mathematical expressions (Equations 3-5), which were programmed in the microcontroller. Finally, it was connected as shown in Fig. 2 and the PID parameters were modified in order to evaluate the behavior of the system, which is shown in Table III.

$$
\begin{aligned}
& A(z) y(t)=B(z) u(t)+C(z) e(t) \\
& A(z)=1-0.3332 z^{\wedge}-1-0.6665 z^{\wedge}-2 \\
& B(z)=0.003627 z^{\wedge}-1-0.003362 z^{\wedge}-2 \\
& C(z)=1+0.4128 z^{\wedge}-1-0.161 z^{\wedge}-2
\end{aligned}
$$


TABLE II. System behavior.

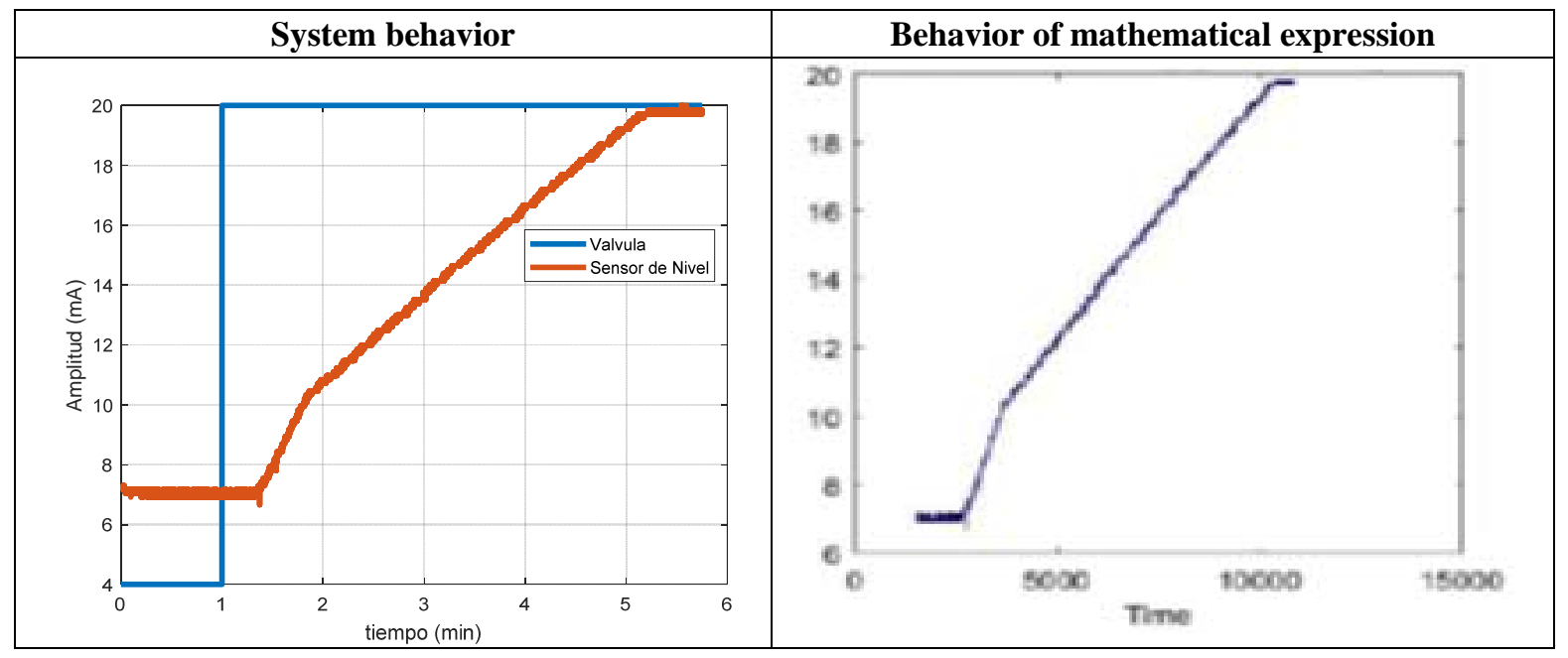

TABLE III. Driver response when simulating the process.

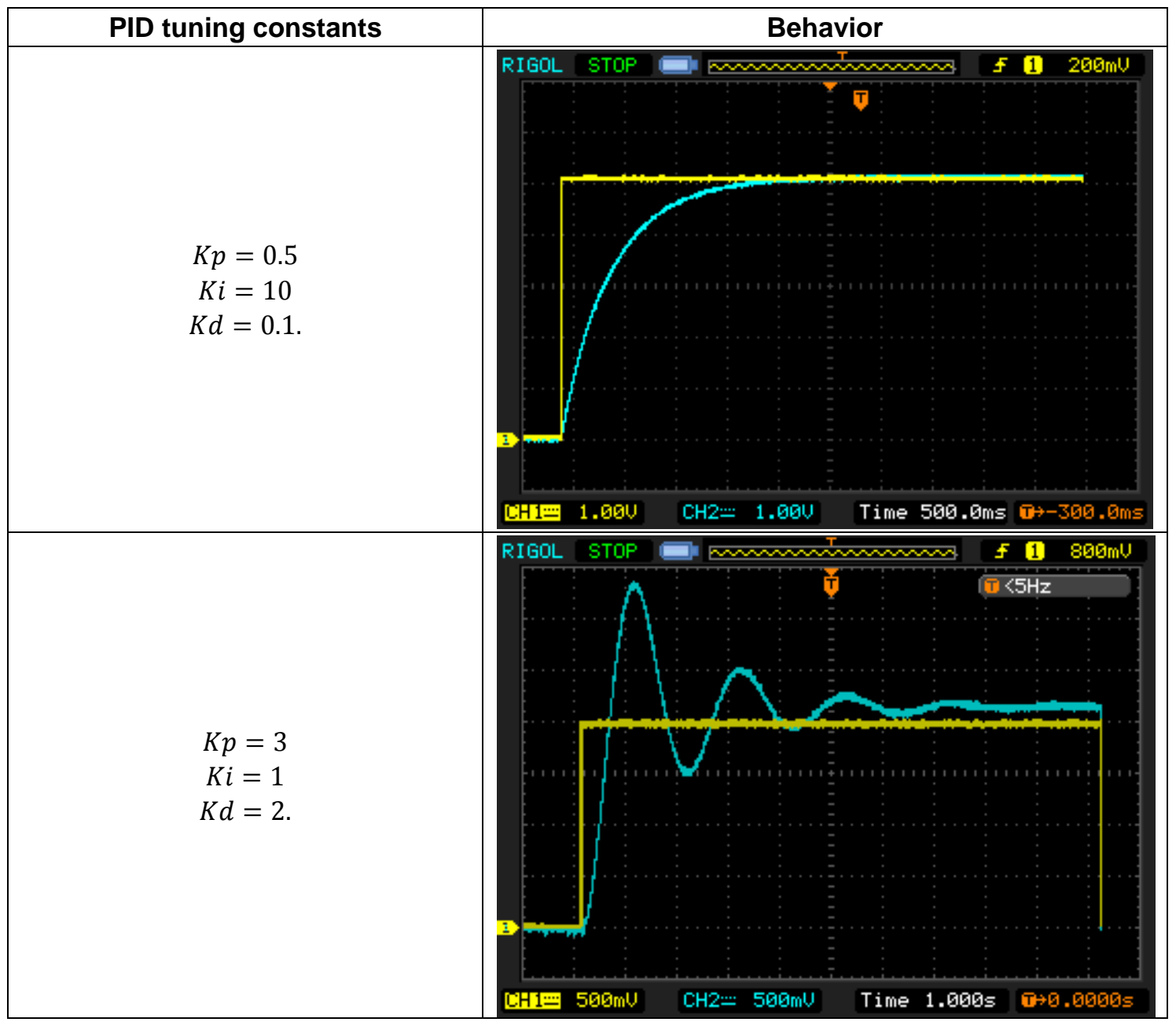

\section{CONCLUSIONS}

The mechanism developed to emulate industrial processes is replicable, since it is based on the amount of process data to imitate a real instrument. An advantage of this method is that it reduces the margin of error that occurs when performing mathematical analyzes in an analytical manner, which benefits the researcher because processes with real and comparable elements are studied. This feature gives an added value to this type of simulations, because, they allow the simulator to find the appropriate set of elements based on parameters of manufacturers and not assumed as it is usually done conventionally. 
The proposed model is relatively simple to implement in a microcontroller, since, compared with other prediction strategies of parameters of a system requires few parameters (3 parameters maximum) as can be seen in the models of neural networks (The number of neurons varies depending on the amount of data) or other types of approximations based on interpolation methods (The polynomial expands according to the number of terms is usually greater than 2). This feature gives the ARIMA models an advantage, since they consume few computational resources when implemented in a processing system.

The identification of systems using the ARIMA models allows us to make an approximation with a margin of error close to $2 \%$. This characteristic from the analytical point of view is quite good, since models with industrial approaches can be obtained from simple mathematical expressions.

\section{ACKNOWLEDGMENT}

This work was supported by the Universidad Distrital Francisco José de Caldas Technological Faculty. The views expressed in this paper are not necessarily endorsed by the University. The authors thank the research group ARMOS for the evaluation carried out on prototypes of ideas and strategies.

\section{REFERENCES}

[1] IEEE Draft Recommended Practice for the Instrumentation and Metering of Industrial and Commercial Power Systems," in IEEE P3001.8 /D8, September 2011, vol., no., pp.1-29, 13 Sept. 2012.

[2] M. Carette et al., "Graduate studies in instrumentation at university of provence," 2009 1st International Conference on Advancements in Nuclear Instrumentation, Measurement Methods and their Applications, Marseille, 2009, pp. 1-2.

[3] Kee-Young Shin and Hwa-Won Hwang, "AROMS: A Real-time Open Middleware System for controlling industrial plant systems," 2008 International Conference on Control, Automation and Systems, Seoul, 2008, pp. 1961-1965.

[4] J. Sevilla and C. Pulido, "Virtual industrial sensors trough neural networks. Demonstration examples in nuclear power plants," IMTC/98 Conference Proceedings. IEEE Instrumentation and Measurement Technology Conference. Where Instrumentation is Going (Cat. No.98CH36222), St. Paul, MN, USA, 1998, pp. 293-297 vol.1.

[5] W. Ikram, S. Petersen, P. Orten and N. F. Thornhill, "Adaptive Multi-Channel Transmission Power Control for Industrial Wireless Instrumentation," in IEEE Transactions on Industrial Informatics, vol. 10, no. 2, pp. 978-990, May 2014.

[6] F. A. Ruslan, K. Haron, A. M. Samad and R. Adnan, "Multiple Input Single Output (MISO) ARX and ARMAX model of flood prediction system: Case study Pahang," 2017 IEEE 13th International Colloquium on Signal Processing \& its Applications (CSPA), Batu Ferringhi, 2017, pp. 179-184.

[7] F.A. Ruslan, R. Adnan, Z.M. Zain, A.M. Samad, "Multiple Input Single Output (MISO) ARX and NARX Model of flood prediction system: A Comparative study", Control System Computing and Engineering (ICCSCE). 2013 IEEE Conference on, pp. 254-255, 2013.

[8] F.A. Ruslan, A.M. Samad, Z.M. Zain, R. Adnan, "Multiple Input Single Output (MISO) ARX and NARX model of flood prediction system: A comparative study", control system computing and engineering (ICCSCE). 2013 IEEE Conference on 2013, pp. 254-255, 2013.

[9] C. Zheng and J. Zhu, "Research on stock price forecast based on gray relational analysis and ARMAX model," 2017 International Conference on Grey Systems and Intelligent Services (GSIS), Stockholm, 2017, pp. 145-148.

[10] M. Haseyama, Y. Aketa and H. Kitajima, "A method for realization of an ARMAX lattice filter," 1995 International Conference on Acoustics, Speech, and Signal Processing, Detroit, MI, USA, 1995, pp. 1705-1708 vol.3.

[11] P. Sahu and S. K. Pradhan, "Multi variable self-tuning PID control of a Twin Rotor MIMO system," 2014 IEEE International Conference on Advanced Communications, Control and Computing Technologies, Ramanathapuram, 2014, pp. 381-385.

[12] Shen Shi Cheng, "Neural-PID visual tracking of moving object," Proceedings of 2011 International Conference on Electronics and Optoelectronics, Dalian, 2011, pp. V1-446-V1-452.

[13] Y. Huang and S. Yasunobu, "A general practical design method for fuzzy PID control from conventional PID control," Ninth IEEE International Conference on Fuzzy Systems. FUZZ- IEEE 2000 (Cat. No.00CH37063), San Antonio, TX, USA, 2000, pp. 969-972 vol.2.

[14] G. Lammel et al., "Next generation pressure sensors in surface micromachining technology," The 13th International Conference on Solid-State Sensors, Actuators and Microsystems, 2005. Digest of Technical Papers. TRANSDUCERS '05, Seoul, South Korea, 2005, pp. 35-36 Vol. 1.

[15] U. Mescheder et al., "MEMS-Based Air Quality Sensor," TRANSDUCERS 2007 - 2007 International Solid-State Sensors, Actuators and Microsystems Conference, Lyon, 2007, pp. 1417-1420. 